\title{
Correlation of Cerebrospinal Fluid Sialic Acid with Various Enzymes in Patients with Meningitis
}

\author{
Nawal Abdullah Murtadha \\ Department of Basic Science, Dentistry College, University of Kirkuk, Kirkuk, Iraq
}

\begin{abstract}
Meningitis is often associated with cerebral compromise which may be responsible for neurological squeal in nearly half of the survivors. Little is known about the mechanisms of Central Nervous System (CNS) involvement in bacterial meningitis. The present study was undertaken to assess the changes in biochemical parameters including glucose, protein, Sialic Acid (SA) and various enzymes like Aspartate Aminotransferase (AST), Alanine Aminotransferase (ALT), Creatine Kinase (CK), Gamma Glutamyltranspeptidase $\gamma$ (GGTP) and Lactate Dehydrogenase (LDH) have been determined in the Cerebrospinal Fluid (CSF) of the meningitis patients $(n=26)$ and compared with control subjects $(n=26)$. Total Protein, CK, SA $(P<0.02)$, AST $(P<0.001)$ and ALT $(\mathrm{P}<0.0001)$ of meningitis patients were significantly higher, whereas, glucose $(\mathrm{P}<0.05)$ and Gamma Glutamyltranspeptidase (GGT) ( $P<0.01$ and $P<0.05$, respectively) were significantly lower than those of the controls. This study suggests that loss of integrity of brain-CSF barriers, sialic acid and enzyme profiles may contribute to the severity and neurological complications of meningitis.
\end{abstract}

Keywords: Cerebrospinal fluids, meningitis, Sialic Acid.

\section{Introduction}

Meningitis is a potentially life-threatening condition. The potential for serious neurological damage or even death necessitates its prompt medical attention and evaluation. Examination of the CSF may provide critically important diagnostic information about the disease. Rapid differentiation of the bacterial and other etiology is important due to its acute complications and long term morbidity especially in children [1, 2]. Sialic Acids are nine-carbon sugars prominently displayed as terminal monosaccharides on surface expressed glycoconjugates of all mammalian cells. Sialic Acids serve key roles in a diverse array of physiological and pathological processes, including organ development, immune regulation, microbial binding, malignancy, and aspects of human evolution [3, 4].

Determination of levels of various enzymes is an established method for diagnosing and assessing the

Corresponding author: Nawal Abdullah Murtadha, Ph.D., research field: clinical biochemistry. severity of many diseases of heart, liver, muscles, prostate, bone and many other organ systems but its role in neurological disorders is yet uncertain.

Brain is a rich source of a variety of enzymes and any injury to brain tissue could similarly result in an increase in activity of these enzymes in cerebrospinal fluid. A simultaneous increase in serum levels will probably depend on integrity of blood brain barrier. If injury is severe enough to disrupt the blood brain barrier, there might be some rise in enzymatic activity in serum [5]. It was in this context that the present study was done to assess the levels of SA and various enzymes like ALT, AST, ALP, CK and LDH in CSF of patients with meningitis and assess the correlation between CSF SA and CSF various enzymes.

\section{Material and Methods}

A prospective hospital based study was done during a period of one year (from 1st November 2013 to 1st November 2015). All patients from one month to 6 years admitted to the Tikrit General Hospital in Tikrit, and Pediatric Hospital in Kirkuk, Iraq with a 
presumptive diagnosis of meningitis were included in the study.

The fluid was withdrawn by lumbar puncture, using a spinal needle No. 20. The patient lies on a hard bench, taking the lateral reclined position and the needle is gently placed above or beneath the forth lumber vertebra. The amount of withdrawn CSF is not fixed, but usually in the range of 1-3 mL. The CSF was examined grossly for appearance and color. Freshly collected specimens were stored at $4{ }^{\circ} \mathrm{C}$. Turbid specimens were centrifuged at 3,000 rpm for 10 minutes before storage. The biochemical parameters such as AST, ALT CK, GGT and LDH levels were done by using fully automated biochemistry analyzer (Cobas Integra 6000 and Cobas C501 from ROCHE diagnostics, Germany) with ready to used kits. CSF SA concentration was determined by thiobarbituric acid method previously described by Warren, L. [6].

Diagnostic criteria included symptoms and signs of fever, headache, meningeal irritation like neck rigidity, sensitivity to bright light and decreased level of consciousness. In infants with nonspecific signs and symptoms fever, irritability, poor feeding and lethargy were considered. Patients were divided into three groups based on their specifications of cerebrospinal fluid samples bacterial meningitis, aseptic (viral) meningitis and no-meningitis. The diagnostic criteria of bacterial meningitis included presence of more than five leukocytes in cubic millimeter of cerebrospinal fluid with dominance of polymorphonuclear cells, low sugar and high protein in cerebrospinal fluid, positive gram staining and positive culture of cerebrospinal fluid. Diagnostic criteria for aseptic meningitis were presence of more than five leukocytes in cubic millimeter of cerebrospinal fluid with the dominance of mononuclear cells, almost normal sugar and protein in cerebrospinal fluid, negative gram staining and culture of cerebrospinal fluid [7]. The CSF samples were divided into two groups.

Group one: Normal control (individual whom suspected as meningitis and the laboratory examination for them were normal), this group includes 26 individuals;

Group two: Meningitis group in whom the clinical and lab features are suggestive of meningitis and they were divided into two groups (bacterial and viral), this group includes 21 patients.

Statistical analysis was performed using SPSS-21 (Statistical Packages for Social Sciences-version 21). Data were tested for normality and Shapiro test confirmed its normality. Unpaired $t$ test was performed to assess significant difference between means. $\mathrm{P}<0.05$ was considered statistically significant.

\section{Results}

The CSF samples were divided into 3 groups according to the results of CSF exam, and the type of meningitis detailed information of all individuals included in this study is summarized in Table 1.

In the current study, the mean CSF, protein and SA level were higher in patients with meningitis than controls $(64.15 \pm 8.08 \mathrm{~g} / \mathrm{L}$ versus $24.8 \pm 4.30 \mathrm{~g} / \mathrm{L})$, and $(39 \pm 6.04 \mu \mathrm{mol} / \mathrm{L}$ versus $10.2 \pm 1.49 \mu \mathrm{mol} / \mathrm{L})$, respectively. Also it shows a significant increase $(\mathrm{P}<$ 0.05) in CK, AST, ALT, LDH and ALP levels in patients with meningitis $(20.99 \pm 1.91 \mathrm{U} / \mathrm{L}),(21 \pm$ $2.55 \mathrm{U} / \mathrm{L}),(43 \pm 8.79 \mathrm{U} / \mathrm{L}),(36.58 \pm 4.79 \mathrm{U} / \mathrm{L})$ and $(13.2 \pm 2.21 \mathrm{U} / \mathrm{L})$ in comparing with control groups $(5$ $\pm 1.01 \mathrm{U} / \mathrm{L}),(6.82 \pm 1.16 \mathrm{U} / \mathrm{L}),(9.4 \pm 1.57 \mathrm{U} / \mathrm{L}),(12$ $\pm 2.56 \mathrm{U} / \mathrm{L})$ and $(2.74 \pm 0.815 \mathrm{U} / \mathrm{L})$ respectively. While, there were significant decreases $(\mathrm{P}<0.01)$ in CSF GGT, glucose level in meningitis group (53 \pm $9.70 \mathrm{IU} / \mathrm{L})(16.54 \pm 3 \mathrm{mg} / \mathrm{dL})$ as compared to control group $(141 \pm 13.2 \mathrm{IU} / \mathrm{L})(61.5 \pm 8 \mathrm{mg} / \mathrm{dL})$.

The results presented there was negative correlation between SA and Total Protein $(r=-0.276)$, glucose $(r$ $=-0.059)$, AST $(\mathrm{r}=-0.041)$, with ALT $\quad(\mathrm{r}=$ -0.425), LDH $(r=-0.267)$, and ADA $(r=-0.324)$ creatine kinase $(r=-0.055)$ in meningitis group. The positive correlation was found between SA and ALP $(r=0.010)$, with GTP $(r=0.016)$ in meningitis group as shown in Table 3. 
Table 1 Demographic characteristic of study population.

\begin{tabular}{llccc}
\hline \multirow{2}{*}{ Group } & \multirow{2}{*}{ Diagnosis } & \multicolumn{3}{c}{ No. of patients } \\
\cline { 3 - 5 } & & Total & Male & Female \\
\hline 1 & Normal (control) & 26 & 15 & 11 \\
3 & Bacterial meningitis & 5 & 4 & 1 \\
4 & Viral meningitis & 16 & 10 & 6 \\
\hline
\end{tabular}

Table 2 Comparison of study variables in two groups studied.

\begin{tabular}{lll}
\hline Parameters & Patient & Control \\
\cline { 2 - 3 } & Mean \pm standard deviation & Mean \pm standard deviation \\
\hline Glucose $(\mathrm{mg} / \mathrm{dL})$ & $16.54 \pm 3$ & $61.5 \pm 8^{*}$ \\
Total protein $(\mathrm{g} / \mathrm{L})$ & $64.15 \pm 8.08$ & $24.8 \pm 4.30^{* *}$ \\
Sialic Acids $(\mu \mathrm{mol} / \mathrm{L})$ & $39 \pm 6.04$ & $10.2 \pm 1.49^{* *}$ \\
CK $(\mathrm{U} / \mathrm{L})$ & $20.99 \pm 1.91$ & $5 \pm 1.01^{* *}$ \\
GGTP $(\mathrm{IU} / \mathrm{L})$ & $53 \pm 9.70$ & $141 \pm 13.2^{* *}$ \\
AST $(\mathrm{U} / \mathrm{L})$ & $21 \pm 2.55$ & $6.82 \pm 1.16^{* *}$ \\
ALT $(\mathrm{U} / \mathrm{L})$ & $43 \pm 8.79$ & $9.4 \pm 1.57^{* *}$ \\
LDH $(\mathrm{U} / \mathrm{L})$ & $36.58 \pm 4.79$ & $12 \pm 2.56^{* *}$ \\
ALP $(\mathrm{U} / \mathrm{L})$ & $13.2 \pm 2.21$ & $2.74 \pm 0.815^{* *}$ \\
\hline
\end{tabular}

${ }^{*} \mathrm{P}<0.05,{ }^{* *} \mathrm{P}<0.01$

Table 3 Correlation of Sialic Acid to other parameters.

\begin{tabular}{ll}
\hline Parameters & Meningitis group $(\mathrm{r})$ \\
\hline AST & $(\mathrm{r}=-0.041)$ \\
ALT & $(\mathrm{r}=-0.425)$ \\
ALP & 0.010 \\
GTP & 0.016 \\
CK & -0.055 \\
Glucose & -0.059 \\
Total protein & -0.276 \\
LDH & -0.267 \\
ADA & -0.324 \\
\hline
\end{tabular}

\section{Discussion}

In the present study, SA levels have been increased significantly in CSF of the patients with meningitis as compared to the controls. These findings are agreement with the results of other studies $[8,9]$. SA (N-acetyl neuraminic acid) is an important nine carbon sugar bound to glycoproteins and glycolipids found in the brain tissue. It exists in nondialysable form, which is released from serum proteins and a dialysable free form derived probably from metabolic activity in CNS. The exact mechanism for increased free $\mathrm{SA}$ in meningitis is not known. However, it could be due to glycoprotein and glycolipid bearing neurons which are rich in sialic acid and contiguous with infected meninges are acted upon by the enzyme neuraminidase elaborated by pyogenic organisms. This enzyme cleaves terminal $\mathrm{N}$-acetyl neuraminic acid from the adjacent sugar of glycoprotein or glycolipids and releases into CSF [10].

Marked significant decrease in glucose concentration was observed in the CSF samples of viral meningitis. Similar results were observed in a study by Lamers, K. J. B. [11] who shows the same results and suggested that the microorganisms in meningitis uses CSF glucose as a source of energy for their metabolism which leads to decrease in its level in CSF. William, E. [12] found that CSF glucose in bacterial meningitis is lower than that in viral one. Ejrnaes, M. et al. [13] have reported the concentration of glucose might normal with elevated protein level in cases of meningitis. Enzyme activity in CSF might be raised because of the reasons: (i) an increased outflow from the serum through an incompetent blood brain barrier; (ii) increased outflow from cells because of cytolysis; (iii) leakage of enzymes from the cells without their obstruction; (iv) decreased removal of 
these enzymes from CSF and (v) increased synthesis and release of these enzymes in CSF as a response to vascular insult. Further studies have shown that when BBB is intact, CSF enzyme activity is unaffected by serum changes. Krogsgaard, A. R. et al. [14] concluded that the neuroaxis was the main source of the enzyme and they were independent of serum levels.

The study revealed a significant increase in the CSF enzyme activities (ALT, AST, ALP, CK and LDH) in meningitis patients as compared to controls. Rise in AST levels cannot be explained only on the basis of disruption of blood brain barrier as normally these enzymes have very low concentration in CSF as compared to serum. The only source from where enzyme could leak into blood is probably the destroyed nervous system, as a result of vascular injury. Various other studies have also suggested rise in serum and CSF AST levels and attributed it to necrosis or anoxic injury to brain tissue $[5,15]$. Sirkis, I. [16] has reported that the activity of the CSF aminotransferases rises during the acute period of meningitis, but higher in meningococcal and tuberculous meningitis than in the viral one. However, no substantial change was observed in blood serum enzymes. Sarojini, B. L. et al. [17] have reported that the studies on CSF-enzymes such as lactate dehydrogenase, CK, Isocitrate dehydrogenase and transaminases (ALT, AST) plays a critical role in the differential diagnosis of meningitis. Sharma, N. et al. [18] have reported that the enzyme activity in CSF was appreciably less than serum in normal subjects as well as in majority of disorders of central nervous system. However, different enzyme activities such as LDH, GGT and CK are elevated in CSF samples of various neurological conditions including infections. In the present study, GGT levels have been increased significantly in CSF of the patients with meningitis as compared to the controls. These findings are agreement with the results of other studies [18, 19], while in agreement with Purna, C. D. [20], who showed significant change of GGTP in meningitis.
A significantly high level of ALP may be due to the drug treatment. Many research reports suggest that the prognosis for meningitis patients could not establish on the basis of enzymatic activity alone, but depends on several factors. The authors conclude that biochemical profile of CSF variables have shown the effectiveness of rapid and definite tests for meningitis and treatment. Marked increase was observed in CSF protein levels in meningitis patients as compared to controls. According to Rotbart, H. A. [21], although the examination of total CSF protein has been found useful in the diagnosis of some neurological disorders such as meningitis, it could not help to any great extent in the diagnosis or differential diagnosis of other diseased conditions. However, this increase in protein level is due to the increased membrane permeability which may lead to increase CSF enzymes proportionately, helping in the differential diagnosis of meningitis [22]. In the current study, LDH levels have been increased significantly in CSF of the patients with meningitis as compared to the controls. This was similar to observation made by Kepa, L. et al. [23]. Sharma, et al. [18] and Knight, J. A. et al. [24].

\section{Conclusion}

The results of this study may have clinical and research implications. It may contribute to further understanding of factors involved in the pathogenesis of brain compromise in bacterial meningitis. It seems to be multifactorial and not due to direct infection of CSF. Increase in the CSF SA, ALT, AST, ALP, CK and LDH were found to be useful in the diagnosis and more accurate treatment for the patients with meningitis.

\section{References}

[1] Tunkel, A. R., and Michael, S. W. 2010. Acute Meningitis. Philadelphia: Churchill Livingstone/Elsevier.

[2] Roos, K. L., Tyler, K. L. 2008. Meningitis. New York: MC Graw Hill.

[3] Chen, X., and Varki, A. 2010. "Advances in the Biology and Chemistry of Sialic Acids." ACS Chem. Biol. 5: 163-176. 
[4] Varki, A. 2010. "Colloquium Paper: Uniquely Human Evolution of Sialic Acid Genetics and Biology." Proc. Natl. Acad. Sci. 107 (2): 8939-8946.

[5] Parakh, N., Gupta, H. L., and Jain, A. 2002. "Evaluation of Enzymes in Serum and Cerebrospinal Fluid in Cases of Stroke." Neurol India 50: 518.

[6] Warren, L. 1971. "The Thiobarbituric Acid Assay of Sialic Acids." J. Biol. Chem. 234: 1971-5.

[7] Shikha, S., and Pradeep Kumar, D. 2014. "Evaluation of CSF Ferritin as an Early Marker for Differentiating Meningitis in Pediatric Patients.” Bali Medical Journal 3 (2): 65-68.

[8] Sharma, J. K. 1989. "Cerebrospinal Fluid Free N-acetyl Neuraminic Acid Levels: A Diagnostic and Prognostic Tests in Meningitis." JAPI 37 (12): 757-759.

[9] Kumar, A. 1984. "CSF and Serum Sialic Acid Levels in Pyogenic Meningitis.” Ind. J. Med. Res. 79: 647-651.

[10] Kulkarni, S. P., Mallikarjuna, C. R., Jayaprakash Murthy, D. S. 2006. "Cerebrospinal Fluid Sialic Acid and Aspartate Transaminase Levels in Meningitis." Indian Journal of Clinical Biochemistry 21 (1) 185-188.

[11] Lamers, K. J. B., and Wevers, R. A. 1995. "Cerebrospinal Fluid Diagnostics: Biochemical and Clinical Aspects." Klin. Biochem. Metab. 3: 63-75.

[12] William, E., Hathaway Wiliam, W., Hay, J., and Jessie, R. 2000. "Groothwis and John Paisley, Bacterial Meningitis." Current Textbook of Pedaitrics Diagnosis and Treatment 11 (22): 726-27.

[13] Ejrnaes, M., Filippi, C., Martinic, M., Ling, E., Togher, L., and Crotty, S. 2006. "Resolution of a Chronic Viral Infection after Interleukin-10 Receptor Blockade.” J. Exp. Med. 203: 2461-2472.

[14] Krogsgaard, A., and Ouaade, R. 1963. "Glutamic Acid Oxaloacetic Acid Transaminase in Spinal Fluid in Infectious Diseases of Central Nervous System." Acta Neurol Scand 39: 154-60.

[15] Nand, N., Gupta, S., and Sharma, M. 1987. "Evaluation of
Enzymes in Serum and CSF in Cases of Cerebrovascular Accidents." Angiology 38: 750-755.

[16] Sirkis, S. I. 1982. "Serum and Cerebrospinal Fluid Enzyme Spectra in Meningitis and Their Differential Diagnostic Value." J. Neuropathi Psychiatrists CC Korsakov 82 (2): 193-7.

[17] Sarojini, B. L., and Ramakrishnan, S. 1991. "Enzymes in Biofluids for Diagnosis of Diseases." Indian Journal of Clinical Biochemistry 6: 143-148.

[18] Sharma, M., and Nand, N. 2006. "Evaluation of Enzymes in Pyogenic and Tuberculous Meningitis." J. Assoc. Physicians India 54: 118-21.

[19] Nand, N., Sharma, M., Saini, D. S., and Khosla, S. N. 1992. "Gamma Glutamyl Transpeptidase in Meningitis." J. Assoc. Physicians India 40 (3): 167-69.

[20] Purna, C. D., and Debasish, P. 2014. "Role of CSF, CK, LDH, GGTP Enzyme Levels in Diagnostic and Prognostic Evaluation of Meningitis." Journal of Clinical and Diagnostic Research 8 (7): 19-22.

[21] Rotbart, H. A. 1997. "Viral Meningitis and the Aseptic Meningitis Syndrome." In Infections of the Central Nervous System, edited by Scheld, W. M., Whitley, R. J., and Durack, D. T. New York: Raven Press.

[22] Almeida, F. J., Lopes, C. R., Arnoni, M. V., and Berezin, E. N. 2007. "Streptococcus Pyogenes Meningitis in Children, Report of Two Cases and Literature Review." Brazil J. Infect Dis. 11: 375-377.

[23] Kepa, L., Oczko-Grzesik, B., and Błedowski, D. 2006. "Evaluation of Cerebrospinal Fluid and Plasma Lactate Dehydrogenase Activity in Patients with Purulent, Bacterial Meningoencephalitis.” Przegl Epidemiol 60 (2): 291-98.

[24] Knight, J. A., Dudek, S. M., and Haymond, R. E. 1981. "Early (Chemical) Diagnosis of Bacterial Meningitis-Cerebrospinal Fluid Glucose, Lactate, and Lactate Dehydrogenase Compared." Clin. Chemistry 27 (8): 1431-34 